\title{
A simple and efficient micrografting method for stably transformed Nicotiana attenuata plants to examine shoot-root signaling
}

\author{
Variluska Fragoso', Hannah Goddard², lan T Baldwin and Sang-Gyu Kim*
}

\begin{abstract}
To adjust their development to the environment, plants rely on specific signals that travel from shoot to root and vice versa. Here we describe an efficient micrografting protocol for Nicotiana attenuata, a useful tool for identifying these signals and understanding their functions. Additionally we analyzed transcript accumulation profiles of scions and rootstocks of grafts performed with wild-type and stably transformed N. attenuata. Our results are consistent with the source-to-sink movement of an sRNA silencing signal.
\end{abstract}

Keywords: Grafting, Nicotiana attenuata, root and shoot signaling, systemic signals

\section{Background}

Many studies have shown that plants use long-distance or systemic signals to coordinate and adjust their growth. These signals convey messages throughout the whole plant, from sensor to effector tissues or organs, and they seem to operate with great specificity which can depend not only on the message itself but also on the spatial and temporal scales over which they act [1]. One of the interesting examples of long-distance signaling in plants is activated by herbivore attack and results in the production of a complex bouquet of plant defenses. Plants are capable of priming defenses systemically in tissues that are distal to the sites of attack, suggesting that an herbivory alert signal is transmitted from attacked to unattacked leaves and roots [2].

Grafting has provided important insights into the study of these systemic wound signals in plants. Grafting a JA biosynthesis mutant with a JA response mutant clearly showed that the production of jasmonic acid (JA) in damaged leaves and the perception of JA by distal leaves are necessary for inducing systemic responses [3]. Moreover, the de novo biosynthesis of JA in systemic leaves was further determined not to be required for the systemic transmission of the wound signals [4]. This

\footnotetext{
* Correspondence: skim@ice.mpg.de

'Department of Molecular Ecology, Max-Planck-Institute for Chemical

Ecology, Hans-Knöll-Straße 8, D-07745 Jena, Germany

Full list of author information is available at the end of the article
}

research focused on the long-distance communication within shoots, and there remains much to be learned about the role of roots in the production and propagation of these important signals throughout the plant that mediate ecological interactions [5].

A wild tobacco, Nicotiana attenuata has been studied in plant-herbivore interaction in its natural habitat, the Great Basin Desert of Utah. Several local and systemic defense responses are induced in $N$. attenuata during herbivore attack. Defense traits, which include trypsin proteinase inhibitors (TPI) and specific volatiles, such as trans- $\alpha$-bergamotene produced by terpene synthases (TPS), are increased in $N$. attenuata when this plant is attacked by herbivores or elicited by herbivore-specific elicitors [6-8]. A notable example is of nicotine, which requires the activity of putrescine $N$-methyltransferase (PMT) in the roots for its synthesis and accumulates the alkaloid in leaves and other above-ground parts of tobacco plants [9]. This alkaloid restrains the consumption of $N$. attenuata's leaves by its attackers, even by Manduca sexta, a specialist and nicotine-tolerant herbivore [10]. The identity of the signal which travels from attacked leaves and activates nicotine production in the roots, and whether this signal is the same that activates other systemic defenses in undamaged leaves are questions yet to be addressed [1].

Research in plant ecology has been greatly enhanced by the manipulation of gene expression in stably
C Biomed Central

() 2011 Fragoso et al; licensee BioMed Central Ltd. This is an Open Access article distributed under the terms of the Creative Commons Attribution License (http://creativecommons.org/licenses/by/2.0), which permits unrestricted use, distribution, and reproduction in any medium, provided the original work is properly cited. 
transformed plants. Isogenic lines expressing either low or high levels of transcripts for a particular gene provide a powerful tool to dissect its organism-level function and fitness consequences of the gene under real-world conditions [11]. However, one drawback of such genetic changes is when the transgene is driven by nonspecific promoters which therefore ectopically express transgenes or silencing constructs, preventing tissue- or organ-specific manipulations.

Here, we describe a simple and highly efficient micrografting protocol for $N$. attenuata, and characterize the influence of the grafting procedure on plant growth. Given that the graft junction is located between the shoots and roots of grafted plants, this technique allows for the independent manipulation of the below- and above-ground parts and the study of their interplay in $N$. attenuata for defense. Our results represent the first step towards the study of shoot-to-root interplay by micrografting in N. attenuata.

\section{Results}

Simple micrografting method for $N$. attenuata has a high success rate

Preliminary cleft grafting experiments with adult plants of $N$. attenuata resulted in low success rate (approximately $10 \sim 15 \%$ ). Therefore, we tested a method using plants in an earlier phase of development. One-week-old seedlings (approximately $2 \mathrm{~mm}$ in height) of different Nicotiana genotypes grown on agar plates were excised below the apical meristem (Figure 1A, B) to prevent adventitious rooting [12]. With a stereomicroscope and the seedlings lying horizontally on the media, the scion of one seedling was placed as close as possible to the rootstock of another. To stabilize the contact between scions and rootstocks, small blocks of agar were placed over the junction of the grafted seedlings. Five days after grafting, a visually apparent connection between the combined parts was observed, surrounded by feeble callus growth (Figure 1B).

Micrografting efficiency varied dramatically among different species of Nicotiana (Figure 1C). N. attenuata proved to be the most suitable among the species tested for this procedure, with an $80 \%$ success rate, as scored one week after grafting. The lowest grafting success rate was observed with $N$. benthamiana (ca. 1\%) in which scions tended to produce roots under these in vitro grafting and growth conditions.

\section{Micrografting does not affect $N$. attenuata growth and development}

Growth parameters of scions and rootstocks of grafted WT/WT $N$. attenuata plants (chimeras are named in a shoot/root manner throughout this paper) were compared weekly to intact WT individuals and no significant

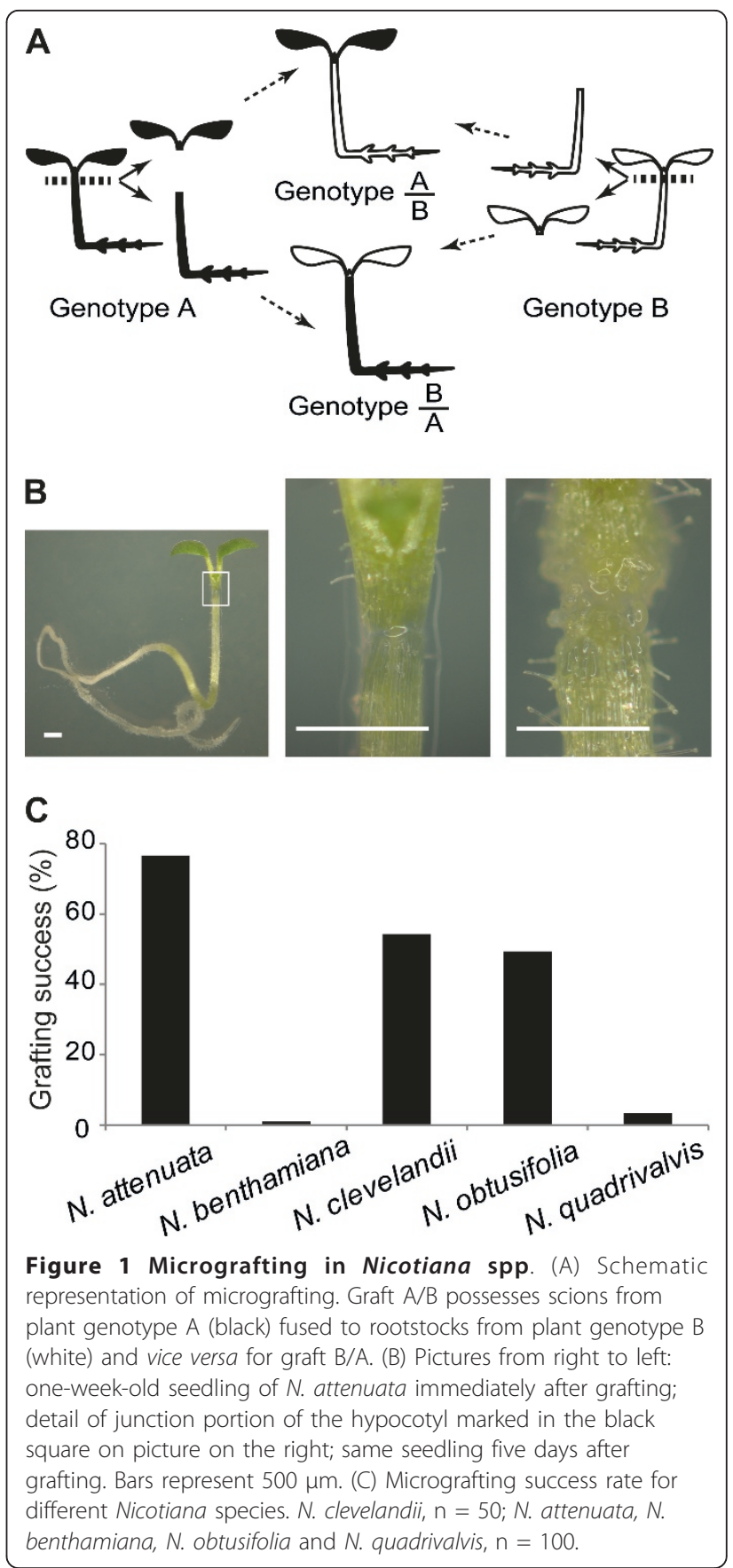

differences were observed (Figure 2). Rosette diameter and height (Figure 2A, B) as well as the length of the longest leaf (data not shown) of grafted WT/WT plants were of similar sizes compared to intact WT plants. The same was also true for biomass comparisons: shoot (Welch's $t$-test; $p=0.34$ ) and root (Welch's $t$-test; $p=$ $0.14)$ dry mass were similar in intact versus grafted plants (Figure 2A). Comparisons of the number of flowers (Welch's $t$-test; $p=0.94$ ) (Figure 2B), capsules (Welch's $t$-test; $p=0.17$ ) and seeds per capsule (Welch's 


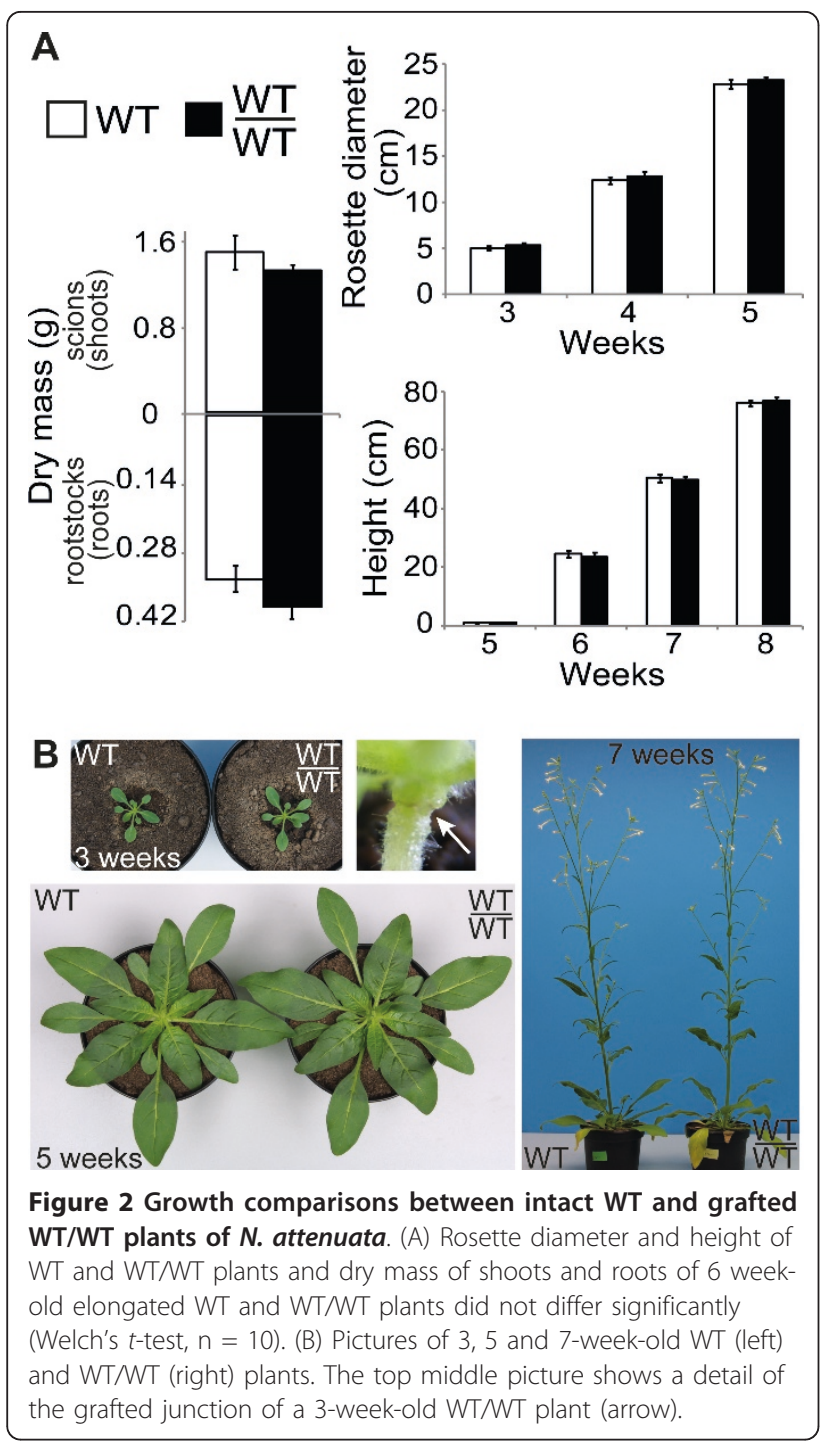

$t$-test; $p=0.31$ ) (data not shown) were also not significantly different.

The graft junction of three-week-old grafted plants was located just below the rosette (Figure 2B, top middle picture). This means that for this work, scions refer to whole shoot or above-ground portions of grafted plants, while rootstocks refer to the entire root system.

\section{Inverted repeat construct of scions affects the transcript accumulation in WT rootstocks}

The main reason for establishing a grafting method for $N$. attenuata is to manipulate defense-related shoot-root signaling in lines already developed by our group (Additional file 1). Such stably transformed plants harboring constructs designed either to silence (antisense - as or inverted repeat - ir) or overexpress (ov) a particular gene are the main approaches by which gene function can be studied in $N$. attenuata system. However, the value of micrografting is mainly limited by the transmission of signals through the graft site [13]. Therefore, to validate the utility of the grafting method using these lines, we verified whether the transcript accumulation of WT scions and rootstocks was altered by their transgenic grafted counterpart. We made use of TPI and $P M T$ genes as innate reporters of the up- and downward spread of silencing since the endogenous expression of these target genes in N. attenuata are particularly useful for this purpose: TPI is mainly expressed in shoots and $P M T$ in roots [14]. We further investigated if the ectopically overexpressed (ov) mRNA of a foreign gene could also be transmitted to WT scions or rootstocks. Importantly, these lines are not morphologically distinguishable from WT $N$. attenuata plants $[7,10,15]$, hence the effects of grafting on plant growth of these transgenic lines were comparable to those observed in grafts performed with WT plants (Figure 2A, B).

As expected [16], accumulation levels of TPI transcripts in leaves of irTPI plants (Figure 3A) were found to be dramatically reduced to below $1 \%$ of WT TPI transcript levels (Fisher's PLSD test; $p<0.0001$ ). Likewise, $P M T$ transcript levels in roots of irPMT plants (Figure 3B) were also found to be reduced to less than $5 \%$ of those found in the roots of WT plants (Fisher's PLSD test; $p<0.01$ ), as previously characterized by Steppuhn et al. [10]. These significant differences found in transcript levels of TPI and PMT of intact plants were maintained when comparing grafted irTPI/irTPI and irPMT/irPMT plants to grafted WT/WT plants, respectively. irTPI/irTPI and irPMT/irPMT plants yield only $0.8 \%$ and $3 \%$ of $T P I$ and $P M T$ transcript levels of WT/WT plants, respectively (Figure 3A, B).

Leaves of irTPI/WT grafts (Figure 3A) failed to accumulate transcripts of TPI and didn't differ significantly from leaves of intact irTPI and grafted irTPI/irTPI plants (Fisher's PLSD test; $p>0.98$ ). In addition, TPI transcript levels of scions of WT/irTPI plants (Figure 3A) didn't significantly differ from those found in WT or grafted WT/WT leaves (Fisher's PLSD test; $p=0.25$ and $p=0.37$, respectively). Roots of WT/irPMT grafts (Figure $3 \mathrm{~B}$ ) expressed similarly low levels of $P M T$ transcripts when compared to the roots of irPMT and irPMT/irPMT grafted plants (Fisher's PLSD test; $p=$ 0.92 and $p=0.85$, respectively). However, the same low levels of PMT transcript (Figure 3B) were found in WT rootstocks of irPMT/WT plants, which didn't differ significantly from irPMT and irPMT/irPMT (Fisher's PLSD test; $p=0.94$ and $p=0.86$, respectively).

Transcript levels of TPS in shoot or roots of nongrafted ovTPS plants (Figure 3C) didn't differ significantly from those found in scions or rootstocks of grafted ovTPS/ovTPS (Fisher's PLSD test; $p=0.29$ and $p$ $=0.09$, respectively). In addition, levels of TPS 


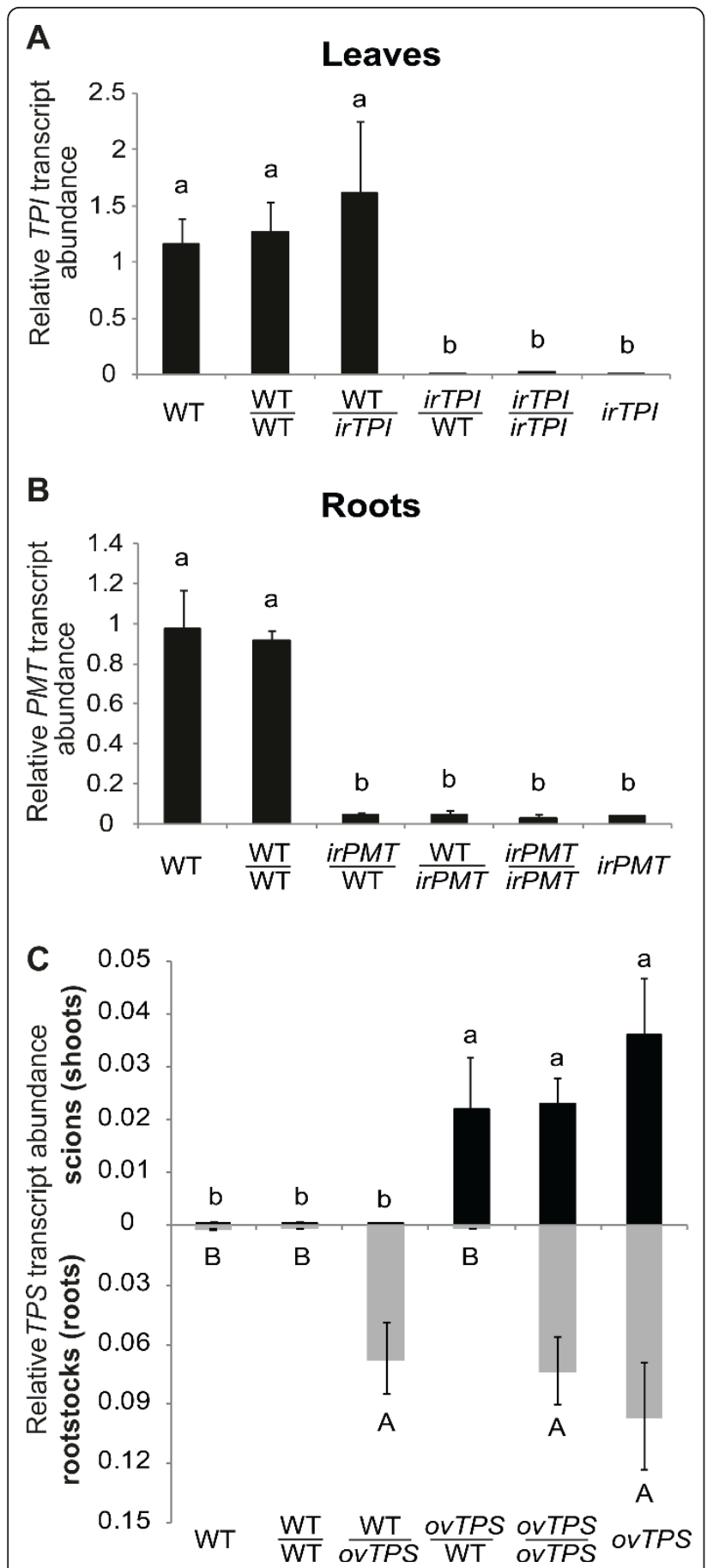

Figure 3 Molecular analysis of leaves and roots of grafted plants transformed with different RNAi and overexpression constructs. (A) TPI (normally expressed only in scions) transcript levels of leaves (scions) of intact or grafted WT and irTPI-silenced plants. (B) PMT (normally expressed only in roots) transcript levels of roots (rootstocks) of intact or grafted WT and irPMT-silenced plants. (C) TPS transcript levels of leaves (scions) and roots (rootstocks) of intact or grafted WT and ovTPS-overexpressing plants, in which a TPS from Z. mays was ectopically expressed behind a 355 promoter. Bars sharing same letters do not significantly differ (Kruskal-Wallis test followed by Fisher's PLSD, $n=5$ ). transcripts in scions of WT/ovTPS and rootstocks of ovTPS/WT (Figure 3C) resembled those of WT or WT/ WT plants (Fisher's PLSD test; $p>0.96$ ).

\section{Discussion}

As previously described for other species $[12,17,18]$, including $N$. benthamiana $[19,20]$, here we describe a simple and highly efficient micrografting protocol for $N$. attenuata with transgenic lines. Our data support the employment of $N$. attenuata grafts that combine WT genotype either to ov- or ir-transgenic lines, this latter only when used as rootstocks, given the altered transcript accumulation of WT roots promoted by silenced shoots. The motivation to adopt this well-known grafting method for $N$. attenuata is grounded in the several layers of inter-related plant defenses that have described in N. attenuata. Systemic signals produced upon herbivore attack enhance the indigestibility of $N$. attenuata leaves by augmented TPI activity [8] and increase its toxicity by producing nicotine [10] and diterpene glucosides [21]. Systemic defense signals are also involved in recruiting predators to attacking herbivores by producing volatiles that betray the location of the herbivores on attacked plants $[6,22]$, as well as in reallocation of energy to roots that are later remobilized for reproduction and thereby enhances a plant's tolerance of herbivore attack [23]. In addition, it is known that $N$. attenuata can change its flower opening time in order to recruit new pollinators that do not have larval stages that are herbivores of the plant and thereby reduces future herbivore loads [24]. Taken together, the establishment of micrografting method for $N$. attenuata will allow us to examine the systemic signals and gene function in above- versus below-ground parts of a plant in an ecological context.

Meristematic activity in the graft junction is an important determinant of attaining high grafting success rates [25]. The reason why previous attempts of cleft grafting with adult $N$. attenuata plants resulted in low success rate may be due to poor callus formation in stem tissues. The hypocotyls of germinated seeds have proved to be the most reliable explant to induce meristematic activity for tissue culture and regeneration of $N$. attenuata [26] and this tissue's particularly high meristematic activity appears also to be the reason behind the success of the of the grafting procedure described here (Figure 1C).

Other than its simplicity and high efficiency, a major advantage of this method for $N$. attenuata is that its impact on a plant's adult life is expected to be minor because the grafting takes place in an early phase of plant development. Only five to six days after grafting, completely healed and healthy grafted seedlings are obtained (Figure 1B), which do not show morphological 
or fitness compromises when compared to intact WT $N$. attenuata plants at later stages in development (Figure 2 ). Moreover, the wounding inflicted by the grafting procedure itself could potentially lead to activation of defense related traits such as augmented TPI expression or nicotine accumulation $[27,28]$. However TPI and PMT transcript accumulation levels of five-week-old WT/WT resembled those of intact WT $N$. attenuata plants (Figure 3A, B). In addition, given that the graft junction is established at the shoot-root interface, this protocol allows for the manipulation of a larger longdistance signaling system in plants, rather than only within shoots [29].

Recent molecular studies suggest the graft hybridization can occur by the exchange of genetic material among neighboring cells and across the graft junction [30-32]. However, as reported for $N$. benthamiana, micrografted roots harboring 35S-derived RNAi constructs were unable to promote silencing of its target in nonsilenced shoots [19]. To validate the micrografting method for $N$. attenuata with transgenic lines, transmission of the silencing effect in rootstocks to scions or vice versa should be examined. Our data are consistent with the lack, or very weak upward transmission of the silencing signal (Figure 3A). On the other hand, when irPMT shoots were grafted onto WT roots of one-weekold seedlings, the roots' ability to accumulate PMT transcripts in later developmental stages was reduced (Figure $3 \mathrm{~B}$ ), consistent with the concept of source-to-sink facilitated movement of sRNA silencing signals [32]. Regardless of the molecular mechanism underlying the spread of the silencing from shoot to root observed in irPMT/WT grafts, these data suggest a limitation to the use of grafts consisting of ir construct-derived transgenic lines scions and WT rootstocks for addressing ecological questions.

Roots are thought to play a role in the control of developmental processes of the aboveground parts of plants, such as shoot branching and flowering [12,33]. As for plant defenses, it has been shown that roots of $N$. attenuata account for both plant resistance (e.g. nicotine production, [10]) and tolerance (e.g. changes in withinplant carbon allocation, [23]) to herbivore attack. However, micrografting can further extend our understanding of a plant's below-ground interactions and molecular mechanisms and their final contribution to the whole-plant performance. For instance, the signaling underlying the JA-induced nicotine synthesis in the roots of $N$. attenuata after leaf damage can be investigated by analyzing grafted plants that have WT shoots and roots deficient in JA perception (irCOI1, [34]), synthesis (irLOX3, [35]) or activation (i.e. conversion of JA to its active JA-Ile form, [36]) (Additional file 1). In addition, ethylene is known to attenuate MeJA-induced accumulation of PMT transcripts as well as the production of nicotine [9]. Given the readily available ethylenerelated transgenic lines (ovETR1 and $i r A C O$, [37]) of $N$. attenuata (Additional file 1), it would be interesting to determine whether ethylene biosynthesis or perception in the roots is the limiting step in the regulation of nicotine synthesis upon herbivore attack. The movement of small RNA is also important in long-distance signaling. Silenced lines of RNA-directed RNA polymerase genes (irRdR1, $i r R d R 2$ and $i r R d R 3,[38-40])$ enable us to find small RNAs that move from WT shoot to small RNA-deficient root after herbivore attack. In addition, silenced lines of Dicer-like (DCL) proteins, which will be available soon, are also useful in manipulating the role of small RNA in shoot-root signaling. Finally, scrutiny of field-grown micrografted $N$. attenuata plants displaying markedly differences in performance could lead to the identification of novel root-derived traits that account for plants' Darwinian fitness as well as serve as a complementary approach to the molecular characterization of genes [12]. Therefore we predict that the protocol reported here will be valuable for unraveling potential root-based traits that profoundly affect plant development and fitness.

\section{Conclusions}

Micrografting combined with a collection of available stably transformed lines, especially for a non-model plant such as $N$. attenuata which has no available mutant libraries, represents a key tool to evaluate genefunction in the many developmental and physiological processes that are governed by long-distance signals. The ectopic expression of ov or ir constructs was restrained in WT/ov, ov/WT and WT/ir grafts. Micrografting thus represents an important advance towards organ-specific characterization of gene function and detection of currently unrecognized long-distance signals, particularly focusing on root physiology which determines the relationships between their below- and above-ground parts and the contribution of this rootshoot communication to whole-plant performance. Given the potential of this method in unraveling root and shoot interplay and the hitherto overlooked importance of roots, we envisage that this procedure will be commonly applied to the study of gene function in $N$. attenuata.

\section{Materials and methods}

\section{Plant materials and growth}

$N$. attenuata seeds are derived from an inbred collection from the DI Ranch, Utah [41]. Seeds of additional Nicotiana sp. were kindly supplied by Dr. Verne A. Sisson (Oxford Tobacco Research Station, Oxford, NC) and originated from collections made by Dr. T. H. 
Goodspeed [42]. For TPI (Accession number: AY426751) and PMT (Accession number: AF280402) silencing in $N$. attenuata, inverted repeat constructs (IR) containing either a fragment of the TPI gene (irTPI) or a consensus fragment for the two $N$. attenuata's $P M T$ genes (irPMT) in inverted orientation were used and characterized by Zavala et al. [15] and Steppuhn et al. [10], respectively. As previously described by these authors, these transgenic lines are not morphologically distinguishable from WT plants and show reduced resistance to herbivores. Transcript levels of TPI and PMT are found reduced to below $1 \%$ in irTPI and $18 \%$ in irPMT plants, respectively, of levels found in WT plants. Consistent with the transcript analysis, no TPI activity was detected in leaf tissues of irTPI plants while levels of nicotine were reduced to $15 \%$ in leaves of $\operatorname{irPMT}$ plants [16]. TPS10-overexpressing $N$. attenuata plants (ovTPS) harbor a sense sequence based on the TPS10 gene of maize (Accession number: AY928078), driven by CaMV 35S promoter [7]. The maize TPS10 gene encodes a terpene synthase involved in the herbivoryinduced production of $(E)$ - $\alpha$-bergamotene and $(E)$ - $\beta$-farnesene which are the major components of the volatile blend emitted by attacked plants and function as attractants of herbivore's natural enemies [43]. As for the ir lines included in this study, no morphological or developmental differences between WT and ovTPS are observed [7]. All seeds were sterilized and treated with $0.1 \mathrm{mM}$ gibberellic acid in 1:50 smoke-distilled water

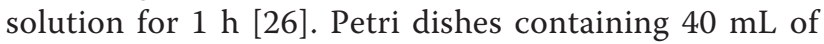
Gamborg's B5 media with minimal organics containing $0.8 \%(\mathrm{w} / \mathrm{v})$ plant agar (Duchefa) were used for germination, manipulation and growth of grafted seedlings. The plates were maintained in growth chambers (Percival, Perry Iowa, USA) at $26 \pm 2^{\circ} \mathrm{C}$, under $16 / 8 \mathrm{~h}$ of light/ dark regime and plants were further transferred to the glasshouse under same conditions when necessary. Grafts were performed in sterile bench under an Olympus SZ51 stereomicroscope. Seedling pictures were obtained using a stereomicroscope equipped with a digital CCD camera (SteREO Discovery.V8, 14 Carl Zeiss Microimaging) and processed with AxioVision LE software (Carl Zeiss 15 Microimaging). Growth parameters were measured weekly $(\mathrm{n}=10)$. Flowers were counted weekly from the $6^{\text {th }}$ until the $8^{\text {th }}$ week of development and the total number of produced flowers was compared. For seed production, mature capsules were collected from plants $(n=5)$ over 5 days starting from the first mature capsule harvested.

RNA isolation and real time quantitative PCR (qPCR)

RNA was extracted from shoots of twenty-day-old or from leaves of five-week-old plants using Tri Reagent [44]. For root RNA extraction, the Tri protocol was modified: $300 \mathrm{mg}$ of ground frozen root material was used and one extra round of centrifugation (12000 g, 15 min) was adopted before addition of chloroform. Extracted RNA was checked on agarose gel and quantified with a NanoDrop ND-1000 spectrophotometer (NanoDrop Technologies, Wilmington, DE, USA). Synthesis of cDNA from $0.5 \mu \mathrm{g}$ of RNA per sample and qPCR analyses were conducted as in Wu et al. [45] using a Mx3005P qPCR system (Stratagene, Santa Clara, CA, USA, http://www.stratagene.com) and qPCR Core Kit for SYBR ${ }^{\circledR}$ Green I (Eurogentec, Seraing, Belgium, http://www.eurogentec.com). Transcript levels were quantified relative to $N$. attenuata elongation factor $1 \mathrm{~A}$ (NaeEF1A) and primers were designed according to Steppuhn et al. [10] and Zavala et al. [15]. All reactions were performed with at least 5 biological replicates.

\section{Statistical analysis}

After verifying data for $t$ - or normal distribution, Welch's $t$-test or Kruskall-Wallis test, followed by Fisher's PLSD as a post-hoc test, were performed using R2.11.1 http://www.R-project.org or StatView5 softwares (SAS Institute, Cary, NC, USA).

\section{Additional material}

Additional file 1: Table 1: List of stably transformed lines of

Nicotiana attenuata. Lines harbor sense (ov), antisense (as) or inverted

repeats (ir) constructs and were created in two different accessions of natural population (Arizona, Az or Utah, Ut) [46-74].

\section{Acknowledgements}

We thank the Max Planck Society for financial support.

\section{Author details}

${ }^{1}$ Department of Molecular Ecology, Max-Planck-Institute for Chemical Ecology, Hans-Knöll-Straße 8, D-07745 Jena, Germany. ${ }^{2}$ MitoSciences, Inc., Eugene, Oregon 97403, USA.

\section{Authors' contributions}

VF carried out the lab work, HG helped with the irTPI and irPMT grafts, ITB and SK conceived the project and oversaw the research. All authors wrote, read and approved the final manuscript.

\section{Competing interests}

The authors declare that they have no competing interests.

Received: 16 September 2011 Accepted: 20 October 2011

Published: 20 October 2011

\section{References}

1. Wu J, Baldwin IT: New insights into plant responses to the attack from insect herbivores. Annu Rev Genet 2010, 44:1-24.

2. Karban R, Baldwin IT: Induced responses to herbivory. Chicago [u.a.]: Univ. of Chicago Press; 1997

3. Li L, Li C, Lee Gl, Howe GA: Distinct roles for jasmonate synthesis and action in the systemic wound response of tomato. Proc Natl Acad Sci USA 2002, 99(9):6416-6421.

4. Li CY, Schilmiller AL, Liu GH, Lee Gl, Jayanty S, Sageman C, Vrebalov J, Giovannoni JJ, Yagi K, Kobayashi Y, et al: Role of beta-oxidation in 
jasmonate biosynthesis and systemic wound signaling in tomato. Plant Cell 2005, 17(3):971-986.

5. Erb M, Lenk C, Degenhardt J, Turlings TC: The underestimated role of roots in defense against leaf attackers. Trends Plant Sci 2009, 14(12):653-659.

6. Kessler A, Baldwin IT: Defensive function of herbivore-induced plant volatile emissions in nature. Science 2001, 291(5511):2141-2144.

7. Schuman MC, Baldwin IT: Asking the ecosystem if herbivory-induced volatile organic compounds have defensive functions. In The integrative role of plant secondary metabolites in ecological systems Volume 1.. 1 edition. Edited by: lanson GR, Dicke M, Hartley SE. Cambridge: Cambridge University Press;

8. Zavala JA, Patankar AG, Gase K, Hui D, Baldwin IT: Manipulation of endogenous trypsin proteinase inhibitor production in Nicotiana attenuata demonstrates their function as antiherbivore defenses. Plant Physiol 2004, 134(3):1181-1190.

9. Winz RA, Baldwin IT: Molecular interactions between the specialist herbivore Manduca sexta (Lepidoptera, Sphingidae) and its natural host Nicotiana attenuata. IV. Insect-Induced ethylene reduces jasmonateinduced nicotine accumulation by regulating putrescine $\mathrm{N}$ methyltransferase transcripts. Plant Physiol 2001, 125(4):2189-2202.

10. Steppuhn A, Gase K, Krock B, Halitschke R, Baldwin IT: Nicotine's defensive function in nature. Plos Biol 2004, 2(8):E217.

11. Gase K, Weinhold A, Bozorov T, Schuck S, Baldwin IT: Efficient screening of transgenic plant lines for ecological research. Mol Ecol Resour 2011, 11(5):890-902.

12. Turnbull CG, Booker JP, Leyser HM: Micrografting techniques for testing long-distance signalling in Arabidopsis. Plant J 2002, 32(2):255-262.

13. Kalantidis $\mathrm{K}$ : Grafting the way to the systemic silencing signal in plants. Plos Biol 2004, 2(8):E224.

14. Saedler R, Baldwin IT: Virus-induced gene silencing of jasmonate-induced direct defences, nicotine and trypsin proteinase-inhibitors in Nicotiana attenuata. J Exp Bot 2004, 55(395):151-157.

15. Zavala JA, Patankar AG, Gase K, Baldwin IT: Constitutive and inducible trypsin proteinase inhibitor production incurs large fitness costs in Nicotiana attenuata. Proc Natl Acad Sci USA 2004, 101(6):1607-1612.

16. Steppuhn A, Baldwin IT: Resistance management in a native plant: nicotine prevents herbivores from compensating for plant protease inhibitors. Ecol Lett 2007, 10(6):499-511.

17. Beveridge CA, Ross JJ, Murfet IC: Branching Mutant rms-2 in Pisum sativum (Grafting Studies and Endogenous Indole-3-Acetic Acid Levels). Plant Physiol 1994, 104(3):953-959.

18. Napoli C: Highly branched phenotype of the petunia dad1-1 mutant is reversed by grafting. Plant Physiol 1996, 111(1):27-37.

19. Kasai A, Bai S, Li T, Harada T: Graft-transmitted siRNA signal from the root induces visual manifestation of endogenous post-transcriptional gene silencing in the scion. PLoS One 2011, 6(2):e16895.

20. Tam DM, Mitter N: Graft-transmissible virus resistance in tobacco (Nicotiana benthamiana). Omonrice 2010, 17:54-64.

21. Heiling S, Schuman MC, Schoettner M, Mukerjee P, Berger B, Schneider B, Jassbi AR, Baldwin IT: Jasmonate and ppHsystemin regulate key malonylation steps in the biosynthesis of 17-hydroxygeranyllinalool diterpene glycosides, an abundant and effective direct defense against herbivores in Nicotiana attenuata. Plant Cell 2010, 22(1):273-292.

22. Allmann S, Baldwin IT: Insects betray themselves in nature to predators by rapid isomerization of green leaf volatiles. Science 2010, 329(5995):1075-1078.

23. Schwachtje J, Minchin PE, Jahnke S, van Dongen JT, Schittko U, Baldwin IT: SNF1-related kinases allow plants to tolerate herbivory by allocating carbon to roots. Proc Natl Acad Sci USA 2006, 103(34):12935-12940.

24. Kessler D, Diezel C, Baldwin IT: Changing pollinators as a means of escaping herbivores. Curr Biol 2010, 20(3):237-242.

25. Pina $A$, Errea P: A review of new advances in mechanism of graft compatibility-incompatibility. Sci Hortic 2005, 106(1):1-11.

26. Kruegel T, Lim M, Gase K, Halitschke R, Baldwin IT: Agrobacterium-mediated transformation of Nicotiana attenuata, a model ecological expression system. Chemoecology 2002, 12(4):177-183.

27. Wu J, Hettenhausen C, Baldwin IT: Evolution of proteinase inhibitor defenses in North American allopolyploid species of Nicotiana. Planta 2006, 224:750-760
28. Ohnmeiss TE, McCloud ES, Lynds GY, Baldwin IT: Within-plant relationships among wounding, jasmonic acid, and nicotine: implications for defence in Nicotiana sylvestris. New Phytol 1997, 137(3):441-452.

29. Schilmiller AL, Howe GA: Systemic signaling in the wound response. Curr Opin Plant Biol 2005, 8(4):369-377.

30. Stegemann S, Bock R: Exchange of genetic material between cells in plant tissue grafts. Science 2009, 324(5927):649-651.

31. Dunoyer P, Schott G, Himber C, Meyer D, Takeda A, Carrington JC, Voinnet O: Small RNA duplexes function as mobile silencing signals between plant cells. Science 2010, 328(5980):912-916.

32. Molnar A, Melnyk CW, Bassett A, Hardcastle TJ, Dunn R, Baulcombe DC: Small silencing RNAs in plants are mobile and direct epigenetic modification in recipient cells. Science 2010, 328(5980):872-875.

33. Abe M, Kobayashi Y, Yamamoto $S$, Daimon Y, Yamaguchi A, keda Y, Ichinoki H, Notaguchi M, Goto K, Araki T: FD, a bZIP protein mediating signals from the floral pathway integrator FT at the shoot apex. Science 2005, 309(5737):1052-1056.

34. Paschold A, Halitschke R, Baldwin IT: Co(i)-ordinating defenses: NaCOI1 mediates herbivore- induced resistance in Nicotiana attenuata and reveals the role of herbivore movement in avoiding defenses. Plant $J$ 2007, 51:79-91.

35. Kallenbach M, Alagna F, Baldwin IT, Bonaventure G: Nicotiana attenuata SIPK, WIPK, NPR1, and fatty acid-amino acid conjugates participate in the induction of jasmonic acid biosynthesis by affecting early enzymatic steps in the pathway. Plant Physiol 2010, 152(1):96-106.

36. Wang L, Allmann S, Wu J, Baldwin IT: Comparisons of LIPOXYGENASE3and JASMONATE-RESISTANT4/6-silenced plants reveal that jasmonic acid and jasmonic acid-amino acid conjugates play different roles in herbivore resistance of Nicotiana attenuata. Plant Physiol 2008, 146(3):904-915.

37. von Dahl CC, Winz RA, Halitschke R, Kuhnemann F, Gase K, Baldwin IT: Tuning the herbivore-induced ethylene burst: the role of transcript accumulation and ethylene perception in Nicotiana attenuata. Plant $J$ 2007, 51(2):293-307.

38. Pandey SP, Baldwin IT: RNA-directed RNA polymerase 1 (RdR1) mediates the resistance of Nicotiana attenuata to herbivore attack in nature. Plant J 2007, 50(1):40-53.

39. Pandey SP, Baldwin IT: Silencing RNA-directed RNA polymerase 2 increases the susceptibility of Nicotiana attenuata to UV in the field and in the glasshouse. Plant J 2008, 54(5):845-862.

40. Pandey SP, Gaquerel E, Gase K, Baldwin IT: RNA-directed RNA Polymerase3 from Nicotiana attenuata is required for competitive growth in natural environments. Plant Physiol 2008, 147(3):1212-1224.

41. Baldwin IT, Gorham D, Schmelz EA, Lewandowski CA, Lynds GY: Allocation of nitrogen to an inducible defense and seed production in Nicotiana attenuata. Oecologia 1998, 115(4):541-552.

42. Goodspeed TH: The genus Nicotiana. Waltham, MA: Chronica Botanica; 1954.

43. Kollner TG, Gershenzon J, Degenhardt J: Molecular and biochemical evolution of maize terpene synthase 10, an enzyme of indirect defense. Phytochemistry 2009, 70(9):1139-1145.

44. Chomczynski P: A reagent for the single-step simultaneous isolation of RNA, DNA and proteins from cell and tissue samples. Biotechniques 1993, 15(3):532-534, 536-537.

45. Wu JQ, Hettenhausen C, Meldau S, Baldwin IT: Herbivory rapidly activates MAPK signaling in attacked and unattacked leaf regions but not between leaves of Nicotiana attenuata. Plant Cell 2007, 19(3):1096-1122.

46. Kang $J H$, Baldwin IT: Isolation and characterization of the threonine deaminase promoter in Nicotiana attenuata. Plant Science 2006, 171(4):435-440.

47. Kessler D, Gase K, Baldwin IT: Field experiments with transformed plants reveal the sense of floral scents. Science 2008, 321(5893):1200-1202.

48. Halitschke R, Baldwin IT: Antisense LOX expression increases herbivore performance by decreasing defense responses and inhibiting growthrelated transcriptional reorganization in Nicotiana attenuata. The Plant Journal 2003, 36(6):794-807

49. Sime KR, Baldwin IT: Opportunistic out-crossing in Nicotiana attenuata (Solanaceae), a predominantly self-fertilizing native tobacco. BMC Ecology 2003, 3:6. 
50. Halitschke R, Ziegler J, Keinanen M, Baldwin IT: Silencing of hydroperoxide lyase and allene oxide synthase reveals substrate and defense signaling crosstalk in Nicotiana attenuata. The Plant Journal 2004, 40(1):35-46.

51. Lou $Y$, Baldwin IT: Silencing of a germin-like gene in Nicotiana attenuata improves performance of native herbivores. Plant Physiology 2006, 140(3):1126-1136.

52. Kang JH, Wang L, Giri A, Baldwin IT: Silencing threonine deaminase and JAR4 in Nicotiana attenuata impairs jasmonic acid-isoleucine-mediated defenses against Manduca sexta. The Plant Cell 2006, 18(11):3303-3320.

53. Wu JS, Kurten EL, Monshausen G, Hummel GM, Gilroy S, Baldwin IT: NaRALF, a peptide signal essential for the regulation of root hair tip apoplastic $\mathrm{pH}$ in Nicotiana attenuata, is required for root hair development and plant growth in native soils. The Plant Journal 2007, 52(5):877-890.

54. Wang L, Halitschke R, Kang JH, Berg A, Harnisch F, Baldwin IT Independently silencing two JAR family members impairs levels of trypsin proteinase inhibitors but not nicotine. Planta 2007, 226(1):159-167.

55. Rayapuram C, Baldwin IT: Increased SA in NPR1-silenced plants antagonizes JA and JA-dependent direct and indirect defenses in herbivore-attacked Nicotiana attenuata in nature. The Plant Journal 2007, 52(4):700-715.

56. Berger B, Baldwin IT: The hydroxyproline-rich glycopeptide systemin precursor NapreproHypSys does not play a central role in Nicotiana attenuata's anti-herbivore defense responses. Plant, Cell and Environment 2007, 30(11):1450-1464.

57. Steppuhn A, Schuman MC, Baldwin IT: Silencing jasmonate signalling and jasmonate-mediated defences reveals different survival strategies between two Nicotiana attenuata accessions. Molecular Ecology 2008, 17(16):3717-3732.

58. Skibbe M, Qu N, Galis I, Baldwin IT: Induced plant defenses in the natural environment: Nicotiana attenuata WRKY3 and WRKY6 coordinate responses to herbivory. The Plant Cell 2008, 20(7):1984-2000.

59. Rayapuram C, Wu J, Haas C, Baldwin IT: PR-13/Thionin but not PR-1 mediates bacterial resistance in Nicotiana attenuata in nature, and neither influences herbivore resistance. Molecular Plant-Microbe Interactions 2008, 21(7):988-1000.

60. Rayapuram C, Baldwin IT: Host-plant-mediated effects of Nadefensin on herbivore and pathogen resistance in Nicotiana attenuata. BMC Plant Biology 2008, 8.

61. Mitra S, Baldwin IT: Independently silencing two photosynthetic proteins in Nicotiana attenuata has different effects on herbivore resistance. Plant Physiology 2008, 148(2):1128-1138.

62. Meldau S, Wu JQ, Baldwin IT: Silencing two herbivory-activated MAP kinases, SIPK and WIPK, does not increase Nicotiana attenuata's susceptibility to herbivores in the glasshouse and in nature. New Phytologist 2009, 181(1):161-173.

63. Korner E, von Dahl CC, Bonaventure G, Baldwin IT: Pectin methylesterase $\mathrm{NaPME} 1$ contributes to the emission of methanol during insect herbivory and to the elicitation of defence responses in Nicotiana attenuata. Journal of Experimental Botany 2009, 60(9):2631-2640.

64. Berger B, Baldwin IT: Silencing the hydroxyproline-rich glycopeptide systemin precursor in two accessions of Nicotiana attenuata alters flower morphology and rates of self-pollination. Plant Physiology 2009, 149(4):1690-1700.

65. Steppuhn A, Gaquerel E, Baldwin IT: The two alpha-dox genes of Nicotiana attenuata: overlapping but distinct functions in development and stress responses. BMC Plant Biology 2010, 10.

66. Allmann S, Halitschke R, Schuurink RC, Baldwin IT: Oxylipin channelling in Nicotiana attenuata: lipoxygenase 2 supplies substrates for green leaf volatile production. Plant, Cell and Environment 2010, 33(12):2028-2040.

67. Onkokesung N, Galis I, von Dahl CC, Matsuoka K, Saluz HP, Baldwin IT: Jasmonic acid and ethylene modulate local responses to wounding and simulated herbivory in Nicotiana attenuata leaves. Plant Physiology 2010, 153(2):785-798.

68. Kaur H, Heinzel N, Schottner M, Baldwin IT, Galis I: R2R3-NaMYB8 regulates the accumulation of phenylpropanoid-polyamine conjugates, which are essential for local and systemic defense against insect herbivores in Nicotiana attenuata. Plant Physiology 2010, 152(3):1731-1747.

69. Wünsche H, Baldwin IT, Wu J: Silencing NOA1 Elevates Herbivory-Induced Jasmonic Acid Accumulation and Compromises Most of the Carbon-
Based Defense Metabolites in Nicotiana attenuata. Journal of Integrative Plant Biology 2011, 53(8):619-631.

70. Colquhoun TA, Schwieterman ML, Wedde AE, Schimmel BCJ, Marciniak DM, Verdonk JC, Kim JY, Oh Y, Galis I, Baldwin IT, et al: EOBII controls flower opening by functioning as a general transcriptomic switch. Plant Physiology 2011, 156(2):974-984

71. Bonaventure G, Schuck S, Baldwin IT: Revealing complexity and specificity in the activation of lipase-mediated oxylipin biosynthesis: a specific role of the Nicotiana attenuata GLA1 lipase in the activation of jasmonic acid biosynthesis in leaves and roots. Plant, Cell and Environment 2011, 34(9):1507-1520.

72. Kallenbach M, Gilardoni PA, Allmann S, Baldwin IT, Bonaventure G: C(12) derivatives of the hydroperoxide lyase pathway are produced by product recycling through lipoxygenase-2 in Nicotiana attenuata leaves. New Phytologist 2011, 191(4):1054-1068.

73. Stitz M, Gase K, Baldwin IT, Gaquerel E: Ectopic expression of AtMT in Nicotiana attenuata: Creating a metabolic sink has tissue-specific consequences for the jasmonate metabolic network and silences downstream gene expression. Plant Physiology 2011, 157:341-354.

74. Gilardoni PA, Hettenhausen C, Baldwin IT, Bonaventure G: Nicotiana attenuata LECTIN RECEPTOR KINASE1 supresses the insect-mediated inhibition of induced defense responses during Manduca sexta herbivory. The Plant Cell 2011.

doi:10.1186/1746-4811-7-34

Cite this article as: Fragoso et al: A simple and efficient micrografting method for stably transformed Nicotiana attenuata plants to examine shoot-root signaling. Plant Methods 2011 7:34.

\section{Submit your next manuscript to BioMed Central and take full advantage of:}

- Convenient online submission

- Thorough peer review

- No space constraints or color figure charges

- Immediate publication on acceptance

- Inclusion in PubMed, CAS, Scopus and Google Scholar

- Research which is freely available for redistribution

Submit your manuscript at www.biomedcentral.com/submit
C) Biomed Central 